\title{
Electron Mobility Study of Hot-Wall CVD GaN and InN Nanowires
}

\author{
Elena Cimpoiasu, Eric Stern, Guosheng Cheng, Ryan Munden, Aric Sanders, and Mark A. Reed \\ Departments of Electrical Engineering, Biomedical Engineering, \\ and Applied Physics, Yale University, P.O. Box 208284, New Haven, CT 06520, USA
}

Received on 5 December, 2005

\begin{abstract}
A review of the dependence of the electron mobility on the free carrier concentration for gallium nitride and indium nitride nanowires grown using hot-wall chemical vapour deposition is presented. Gallium nitride nanowires exhibit mobilities of $100 \mathrm{~cm}^{2} / \mathrm{Vs}$ to below $1 \mathrm{~cm}^{2} / \mathrm{Vs}$ for carrier concentrations of $10^{19}$ to $10^{20} \mathrm{~cm}^{-3}$. Theoretical estimations and annealing experiments indicate that the nanowires are heavily compensated. Indium nitride nanowires also exhibit high carrier concentrations, of the order of $10^{20}$ to $10^{22} \mathrm{~cm}^{-3}$. For both types of nanowires, mobility decreases with increasing carrier concentration, consistent with transport limited by impurity scattering.
\end{abstract}

Keywords: GaN; InN; Nanowires; Electron transport

\section{INTRODUCTION}

The group III-nitrides and their alloys represent a promising system for semiconducting device applications, especially for photonic devices, because they are direct band gap semiconductors with potential light emission from ultraviolet to infrared. Growth of $\mathrm{GaN}$ and $\mathrm{InN}$ nanowires was reported by a number of groups employing mainly catalyzed vaporliquid-solid (VLS) or catalyst-free vapor-solid (VS) growths ([1], [2] and References therein). One simple, economical and very successful method to grow both types of nanowires is using hot-wall chemical vapor deposition. Understanding the electronic properties of the as-grown nanowires is a crucial step towards their implementation in useful devices. Here we summarize the electric properties of a large number of devices (field-effect transistors) that we built with nanowires grown using this simple process. Both indium nitride and gallium nitride nanowires exhibit high carrier concentrations, with mobilities limited by impurity scattering. In particular, the gallium nitride nanowires appear to grow heavily compensated, as inferred from our theoretical estimates and annealing experiments.

\section{EXPERIMENTAL AND RESULTS}

Gallium nitride and indium nitride nanowires were grown using a hot-wall chemical vapor deposition (CVD) system described elsewhere [3]. The GaN NWs were synthesized via metal-catalyzed ( $\mathrm{Ni}$ or $\mathrm{Fe}$ ) VLS growth on alumina or oxidized silicon substrates. This process involved solid gallium source (either metallic Ga or metallic Ga combined with $\mathrm{Ga}_{2} \mathrm{O}_{3}$ powder), heated to temperatures between 800 and $1100{ }^{\circ} \mathrm{C}$, in ammonia flowing at $2 \mathrm{sccm}$ to $100 \mathrm{sccm}$. This process consistently yielded nanowires with similar structural and electrical properties for similar growth parameters, of high crystalline quality.

Indium nitride nanowires were grown using a catalyst-free method, assumed to follow the vapor-solid route. Growth sources were a mixture of indium and indium oxide, heated to $700{ }^{\circ} \mathrm{C}$ in ammonia flowing at $100 \mathrm{sccm}$. This growth process yielded very large amounts of nanowires on the wall of the furnace.

The as-grown nanowires were subsequently suspended in solution and dispersed on silicon wafers covered with $200 \mathrm{~nm}$ oxide. Optical lithography was chosen to fabricate the dispersed wires in field-effect transistor devices as test probes of the electrical properties. The advantage of using optical lithography as opposed to electron beam lithography is that it allows parallel processing of a large number of NWs and provides a blind testing of their electronic properties. In this way a large number of nanowires were electrically investigated and the effects of different growth parameters on these properties were successfully singled out [3].

Figure 1 shows the dependence of the extracted mobilities $\mu$ as a function of the free carrier concentration $n$. The plot includes three sets of data: the filled circles represent results of a non-optimized typical growth (Growth 1), the squares represent results from best, optimized growth (Growth 2), and the triangles are the best reported results for GaN NWs, grown using a laser-assisted VLS process [4]. The growth parameters of Growth 1 and Growth 2 are listed in Table 1.

TABLE I: Growth Parameters for GaN NWs

\begin{tabular}{lll}
\hline & Growth1 & Growth2 \\
\hline Temperature $\left({ }^{\circ} \mathrm{C}\right)$ & 800 & 950 \\
Ammonia Flow $(\mathrm{sccm})$ & 100 & 2 \\
Substrate & $\mathrm{Si} / \mathrm{SiO}_{2}$ & $\mathrm{Si} / \mathrm{SiO}_{2}$ \\
Gallium Source & $\mathrm{Ga}+\mathrm{Ga}_{2} \mathrm{O}_{3}$ & $\mathrm{Ga}+\mathrm{Ga}_{2} \mathrm{O}_{3}$ \\
Pressure & $1 \mathrm{~atm}$ & $1 \mathrm{~atm}$ \\
Metal Catalyst & $\mathrm{Ni}$ & $\mathrm{Fe}$ \\
\hline
\end{tabular}

As seen from Fig. 1, the mobility exhibits a decreasing behavior with increasing carrier concentration (close to inverse power law). This behavior is consistent with mobility limited by scattering processes on ionized impurities. To quantify this behavior, we have calculated the ionized impurity scattering mobility using a Conwell-Weisskopf formula $[5,6]$ : 


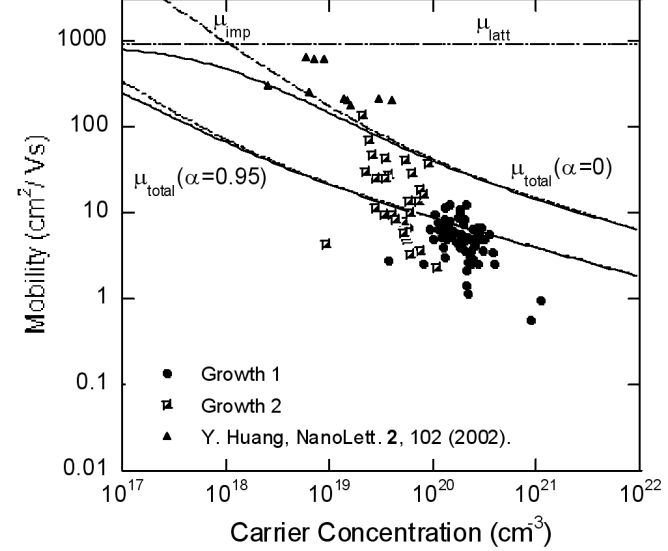

FIG. 1: Mobility versus free carrier concentration for GaN nanowires from Growth 1, Growth 2, and from Ref. [4]. Continuous black lines represent theoretically estimated mobility for compensation levels $\alpha$ $=0$ and 0.95 .

$$
\mu_{i m p}=\frac{64 \sqrt{\pi} \varepsilon^{2}(2 k T)^{3 / 2}}{N_{i} e^{3} \sqrt{m^{*}}}\left\{\ln \left[1+\left(\frac{12 \pi \varepsilon k T}{e^{2} N_{i}^{1 / 3}}\right)^{2}\right]\right\}^{-1}
$$

Here $\varepsilon=8.9 \varepsilon_{0}$ [7] is the semiconductor permittivity, $k$ is the Boltzmann's constant, $T$ is the temperature in Kelvin, $m^{*}=0.2 m_{0}$ [7]) is the effective mass of electrons, and $N_{i}=$ $N_{D}+N_{A}$ is the density of ionized impurities, equal with the sum between ionized donor and acceptor impurities.

Ionized impurity scattering is not the only scattering process for the free carriers. Other mechanisms include phonon (acoustic and optical) scattering, scattering at stacking faults and point defects. However, these mechanisms do not exhibit a relevant $n$ dependence and we account for their effect using a fixed mobility component, $\mu_{\text {latt }}$ [8]. Here we choose $\mu_{\text {latt }}=900 \mathrm{~cm}^{2} / \mathrm{Vs}$ to account for the high values of mobility from Reference [4]. We should note that the exact value of $\mu_{\text {latt }}$ is not particularly relevant for these high carrier concentrations $\left(10^{19}-10^{21} \mathrm{~cm}^{-3}\right)$, as seen from Fig. 1 .

The total mobility $\mu_{\text {total }}$ is then calculated using Matthiesen's rule:

$$
\frac{1}{\mu_{\text {total }}}=\frac{1}{\mu_{\text {imp }}}+\frac{1}{\mu_{\text {latt }}}
$$

Figure 1 shows the estimated total mobility for uncompensated $(\alpha=0)$ and heavily compensated $(\alpha=0.95)$ material (continuous black line, $\left.\alpha=N_{A} / N_{D}\right)$. One can notice that Growth 1 exhibits heavy levels of compensation, while the optimized one lies in between the uncompensated and heavily compensated lines. This indicates that the observed reduction in the carrier concentration is due to a substantial decrease of the donor impurities/defects sites.

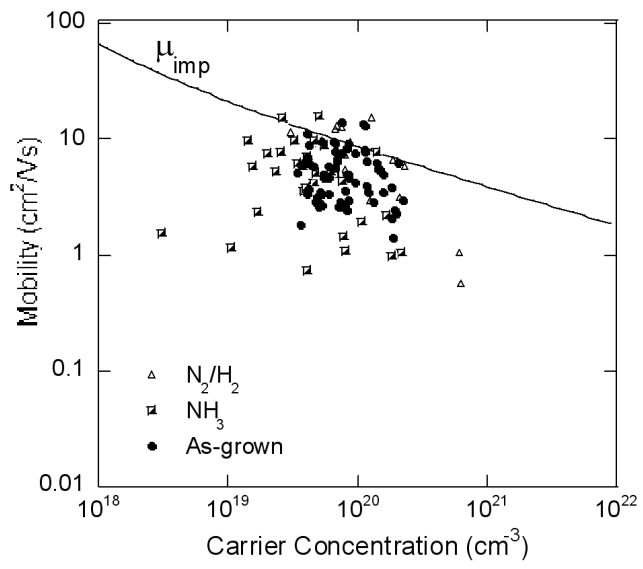

FIG. 2: Mobility versus free carrier concentration for GaN nanowires from as-grown and ammonia or forming gas annealed samples (with growth parameters similar with Growth 1 , except $\mathrm{T}_{\text {growth }}=900^{\circ} \mathrm{C}$ ). Continuous black line represents theoretically estimated mobility for $\alpha=0.95$.

In order to understand more of the origin of these high carrier concentrations, we performed annealing experiments of samples with growth parameters similar to the Growth 1 and Growth 2. If the source of the low mobility/high carrier concentration are nitrogen vacancies and oxygen impurities (as it is wildly speculated in the literature), annealing in conditions that would favor reduction of their concentration would result in improved electrical properties (low $n$, high $\mu$ ). It is conceivable that annealing in ammonia at high temperature for long periods of time would allow diffusion of nitrogen from decomposed ammonia into the nanowires to fill in the nitrogen vacancies. By the same token, annealing in forming gas would extract some oxygen impurities, therefore reduce the concentration of one other important source of $n$-type behavior.

The results of annealing of samples similar to Growth 1 (with $\mathrm{T}_{\text {growth }}=900^{\circ} \mathrm{C}$ ) in flowing ammonia or forming gas (for $4 \mathrm{~h}$ ) are shown in Fig. 2, together with the theoretical estimate of the impurity scattering mobility. The forming gas annealing resulted in virtually no change of the electrical properties of the nanowires (the mean mobility and log carrier concentration for the as-grown were $5.21 \pm 2.85 \mathrm{~cm}^{2} / \mathrm{Vs}$ and $19.89 \pm 0.21$, respectively, while for the one annealed in forming gas were $7.53 \pm 4.53 \mathrm{~cm}^{2} / \mathrm{Vs}$ and $20.09 \pm 0.35$ ). The annealing in ammonia, on the other hand, produced a statistically significant decrease in the carrier concentration, to $19.62 \pm 0.38$, while leaving the mobility virtually unchanged at $5.57 \pm 3.92 \mathrm{~cm}^{2} / \mathrm{Vs}$. The result of annealing in forming gas versus ammonia could be interpreted as indirect evidence for the dominant role of nitrogen impurities versus the oxygen impurities in the nanowires, i.e., presumably lower nitrogen vacancy concentration (due to ammonia anneal) reduced $n$, while presumably lower oxygen concentration did not affect $n$. However, it is hard to draw a definite conclusion regard- 
the reduction in mobility due to impurity scattering. The ex-

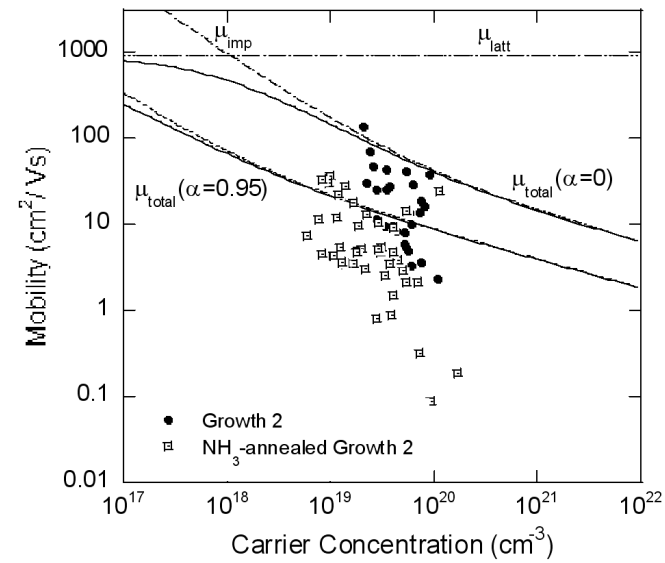

FIG. 3: Mobility versus free carrier concentration for GaN nanowires from as-grown and ammonia-annealed samples from Growth 1 . Continuous black lines represent theoretically estimated mobility for compensation levels $\alpha=0$ and 0.95 .

ing the cause of decreased $n$, especially at these high carrier concentration levels. Indeed, because of the small slope of the $\mu-N_{i}$ dependence and the high device-to-device fluctuations, it is hard to distinguish between reduction of $n$ due to the decrease of the donor sites (which should also increase mobility) or reduction of $n$ due to increase in the compensation levels (accompanied by a decrease of mobility).

In order to confirm the role of ammnonia anneal on nitrogen vacancies/impurities levels, we performed a second anneal using samples from the optimized growth (Growth 2). This result is shown in Fig. 3, where the filled circles represent the properties of the as-grown sample, while the centered squares represent the properties of the $4 \mathrm{~h} \mathrm{NH}_{3}$-annealed sample. It is evident that the annealed sample has significantly lower carrier concentration and mobility, indicative of increased levels of compensation. Thus, we conclude that the reduction of the carrier concentration after annealing in ammonia is due to compensation effects. Although data does not allow concluding remarks about the source of compensation, we can speculate that hydrogen (from the decomposed ammonia) and probably carbon might diffuse inside the nanowires and participate in donor compensation [9].

Theoretical estimates similar to above were performed for the electronic properties of $\mathrm{InN}$ nanowires. Figure 4 shows the mobility dependence on carrier concentration for two similar InN NWs growths, together with published results on epitaxially-grown InN grown on GaN [11-14] and sapphire substrates [11]. The solid curve is the calculated impurity scattering mobility using Eq. 1 , where $\varepsilon=15.3 \varepsilon_{0}$ and $m^{*}=0.11 m_{0}[15]$. The nanowires show high carrier concentrations, of the order of $10^{20}$ to $10^{22} \mathrm{~cm}^{-3}$, but the values of the mobility line up to the results previously reported for the epitaxially-grown films. The theoretical estimate exhibits a reasonable agreement to the data, with an overestimation of

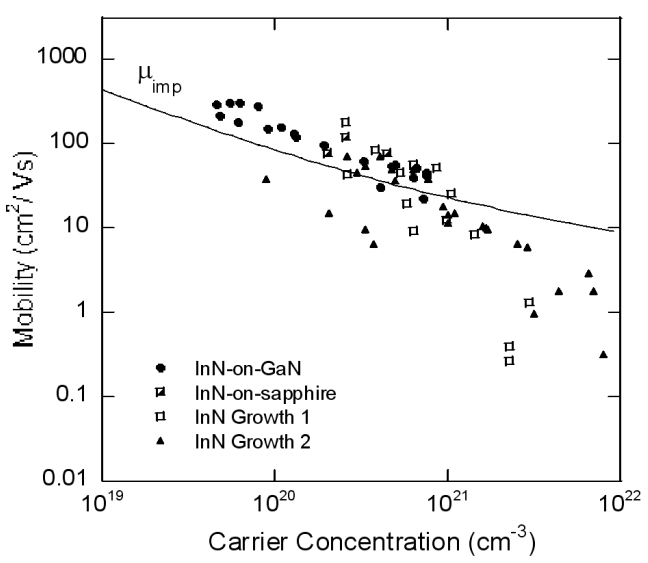

FIG. 4: Scatter plot of mobility vs. carrier concentration for two growth-fabrication runs of InN NWs. Data from bulk epitaxiallygrown InN on sapphire [11] and GaN [11-14] substrates are included. Continuous black line represents theoretically estimated mobility due to ionized impurity scattering $(\alpha=0)$.

perimental points deviate from the theoretical curve for $n$ over $10^{21} \mathrm{~cm}^{-3}$, probably due other competing scattering mechanisms at these high concentration levels.

\section{CONCLUSION}

Gallium nitride and indium nitride were successfully grown using hot-wall chemical vapor deposition (tube furnace) and built into a large number of devices which were used to statistically probe their electronic properties. We found that both types of nanowires exhibit large carrier concentrations, with a decreasing mobility upon increasing free carrier concentrations, consistent with transport dominated by impurity scattering. In the case of the gallium nitride nanowires, using feedback between the growth parameters and electrical characterization, we were successful in decreasing (increasing) the carrier concentration (mobility) by one order of magnitude. Also, simple theoretical estimates and annealing experiments indicate that the gallium nitride nanowires grow heavily compensated.

\section{Acknowledgement}

We would like to thank Dr. Jim Klemic, Dr. Nilay Pradhan, and David Routenberg for useful discussions. This work was partially supported by DARPA through AFOSR, ARO (DAAD19-01-1-0592), AFOSR (F49620-01-1-0358), NASA (NCC 2-1363), by the Department of Homeland Security (graduate fellowship), and by the National Science Foundation (graduate fellowship). 
[1] M. Law, J. Goldberger, and P. Yang, Annu. Rev. Mater. Res. 34, 83 (2004).

[2] G. Cheng, E. Stern, D. Turner-Evans, and M. A. Reed, Appl. Phys. Lett. 87, 253103 (2005). .

[3] E. Stern, G. Cheng, E. Cimpoiasu, R. Klie, S. Guthrie, J. Klemic, I. Kretzschmar, E. Steinlauf, D. Turner-Evans, E. Broomfield, J. Hyland, R. Koudelka, T. Boone, M. Young, A. Sanders, R. Munden, T. Lee, D. Routenberg, and M. A. Reed, Nanotechnology 16, 2941 (2005)

[4] Y. Huang, X. Duang, Y. Cui, and C.M. Lieber, Nano Letters 2, $102(2002)$

[5] S.M. Sze, Physics of Semiconductor Devices, John Wiley \& Sons New York, pg. 28, 1985.

[6] E. Conwell and V.F. Weisskopf, Phys. Rev. 77, 388 (1950).

[7] I.M. Abdel-Motaleb and R.Y. Korotkov, J. Appl. Phys. 97, 093715 (2005).

[8] N. G. Weimann and L. F. Eastman, J.Appl. Phys. 83, 3656
(1998).

[9] We should mention, however, that calculations for the diffusion barrier of hydrogen predict $\sim 3.4 \mathrm{eV}$, a reason for which hydrogen in not very mobile and large amounts of hydrogen are not readily incorporated during growth [10].

[10] J. Neugebauer and C.G. Van de Walle, Appl. Phys. Lett. 68, 1829 (1996).

[11] A. Yamamoto, T. Shin-ya, T. Sugiura, and A. Hashimoto, J. Cryst. Growth 189/190, 461 (1998).

[12] C. R. Abernathy, S. J. Pearton, F. Ren, and P. W. Wisk, J. Vac. Sci Technol. B 11, 179 (1993).

[13] W. A. Bryden, S. A. Ecelberger, and T. J. Kistenmacher, Appl. Phys. Lett. 64, 2864 (1994).

[14] Y. Sato and S. Sato, J. Cryst. Growth 146, 262 (1994).

[15] V.W.L. Chin, T.L. Tansley, and T. Osotchan, J. Appl. Phys. 75, 7365 (1994). 